\title{
Community-wide consequences of sexual dimorphism: evidence from nectar microbes in dioecious plants
}

\author{
AUTHOR(S): \\ Tsuji, Kaoru; Fukami, Tadashi
}

\section{CITATION:}

Tsuji, Kaoru ... [et al]. Community-wide consequences of sexual dimorphism: evidence from nectar microbes in dioecious plants. Ecology 2018, 99(11): 2476-2484

\section{ISSUE DATE:}

2018-11

URL:

http://hdl.handle.net/2433/234585

RIGHT:

Copyright (c) 2018 by the Ecological Society of America 


\title{
Community-wide consequences of sexual dimorphism: evidence from nectar microbes in dioecious plants
}

\author{
Kaoru Tsuji (iD) 1,2,3 And TADAshi Fukami (D) 2 \\ ${ }^{1}$ Center for Ecological Research, Kyoto University, 2-Hirano, Otsu, Shiga 520-2113 Japan \\ ${ }^{2}$ Department of Biology, Stanford University, Stanford, California 94305 USA
}

\begin{abstract}
Intraspecific trait variation is receiving renewed interest as a factor affecting the structure of multi-species communities within and across trophic levels. One pervasive form of intraspecific trait variation is sexual dimorphism in animals and plants, which might exert large effects particularly on the communities of host-associated organisms, but the extent of these effects is not well understood. We investigated whether host-associated microbial communities developed differently in the floral nectar of female and male individuals of the dioecious shrubs, Eurya emarginata and E. japonica. We found that nectar-colonizing microbes such as bacteria and fungi were more than twice as prevalent and, overall, more than 10 times as abundant in male flowers as in female flowers. Microbial species composition also differed between flower sexes. To examine potential mechanisms behind these differences, we manipulated the frequency of flower visitation by animals and the order of arrival of microbial species to nectar. Animal visitation frequency affected microbial communities more greatly in male flowers, while arrival order affected them more in female flowers. These sex-specific effects appeared attributable to differences in how animals and microbes altered the chemical characteristics of nectar that limited microbial growth. Taken together, our results provide evidence that sexual dimorphism can have large effects on the structure of host-associated communities.
\end{abstract}

Key words: competitive release; dispersal; flower; host-associated microbes; nectar bacteria; nectar yeast; pollination; priority effects; sexual difference; symbiosis.

\section{INTRODUCTION}

Over the past decade, there has been increasing recognition that intraspecific trait variation can have a major effect on how ecological communities develop, potentially rivaling the effect of interspecific trait variation (Bolnick et al. 2011, Violle et al. 2012, Lajoie and Vellend 2015). One pervasive and often dramatic form of intraspecific variation is sexual dimorphism in animals and plants. Due to their divergent traits, females and males can interact with heterospecific individuals differently, as demonstrated in competitive (Butler et al. 2007, Wagner et al. 2012), predator-prey (Schoener 1967, Ryan et al. 1982, Sakashita 1992, Sommer 2000, Costantini et al. 2007, Tobler et al. 2008, Kwieciński et al. 2017), plant-herbivore (Agren et al. 1999, Cole and Ashman 2005, Cornelissen and Stiling 2005, Vesakoski et al. 2008, Tsuji and Sota 2010, 2013), plant-pollinator (Temeles et al. 2009, Zhang and He 2017), and plant-pathogen (Agren et al. 1999) interactions. These studies suggest that sex-specific interactions have the potential to shape a range of communities within and across trophic levels.

One type of community that may be particularly prone to the influence of sex-specific interactions is those of hostassociated organisms. When host individuals are sexually dimorphic in the traits that determine their quality as a host, sex-specific differences in host-associated communities could arise. As they assemble into different compositions, host-associated communities can modify female and male host quality differently, causing the communities to diverge

Manuscript received 3 January 2018; revised 21 May 2018; accepted 10 July 2018. Corresponding Editor: Matthias C. Rillig.

${ }^{3}$ E-mail: tsuji@ecology.kyoto-u.ac.jp further in species composition. To date, however, only a small number of cases have been documented, with a nearly exclusive focus on medically relevant animal hosts (Fierer et al. 2008, Markle et al. 2013, Cong et al. 2016, Sylvia et al. 2017, Suzuki et al. 2017, but see Golonka and Vilgalys 2013, Petry et al. 2013, Wei and Ashman 2018). Furthermore, this line of research has often focused on a specific group of organisms, often pathogens (Ågren et al. 1999), rather than taking a broader look at communities. Consequently, the extent to which sexual dimorphism affects hostassociated communities remains poorly understood.

In this paper, we investigate whether host-associated communities develop differently in the floral nectar of female and male plants. Many dioecious plants show sexual dimorphism in the chemical characteristics of floral nectar including the concentration and composition of sugars and other chemicals, which might reflect adaptive evolution towards increased pollination (Baker and Baker 1983, Delph and Lively 1992, Eckhart 1999, Bai et al. 2011, Heil 2011, Nepi et al. 2012). Although rarely considered in the context of sexual dimorphism (Golonka and Vilgalys 2013, Wei and Ashman 2018), the chemical properties of nectar could also affect its quality as a microbial habitat (De Vega et al. 2009, Herrera and Pozo 2010, Pozo et al. 2012, 2015, ÁlvarezPérez and Herrera 2013, Aleklett et al. 2014, Herrera 2014, Vannette and Fukami 2014, Canto et al. 2015, Lievens et al. 2015, Mittelbach et al. 2015). Nectar is often colonized by multiple species of fungi and bacteria via flower-visiting animals, resulting in microbial community assembly in each flower (Lachance et al. 2001, Brysch-Herzberg 2004, Herrera et al. 2009, Belisle et al. 2012). Recent research indicates that two drivers of this community assembly are animal visitation frequency and microbial dispersal history, 
both of which vary among flowers (Peay et al. 2012, Mittelbach et al. 2016, Vannette and Fukami 2017). Combining this previous knowledge with the observation that sugar concentration in nectar tends to be lower in male than female flowers, we developed and tested three hypotheses: nectar microbes are more abundant in male than female flowers (hypothesis 1), and animal visitation frequency (hypothesis 2) and microbial dispersal history (hypothesis 3) have a greater effect on microbial community assembly in male than female flowers. We elaborate on the rationale for these hypotheses in the next section.

\section{Materials And Methods}

Our study system was Eurya emarginata and E. japonica, which are common dioecious shrubs native to much of Japan and other parts of East Asia (Tsuji and Sota 2011, Chung and Chung 2000; Fig. 1a-d). We used E. emarginata plants at two coastal sites in Kushimoto and E. japonica plants at the same wood edge site in Kozagawa as used by Tsuji and Sota $(2010,2013)$, all located in Wakayama Prefecture in Japan (Fig. 1e). Their flowers are visited by a diversity of insects (Fig. 1a-d), including flies, midges, and bees. Previously, we reported that some of these insects were sometimes more frequently found around male plants than around female plants, but the difference was small and inconsistent between years (Tsuji and Ohgushi 2018). Floral longevity is similar between plant sexes (mean of 9 and $11 \mathrm{~d}$ for female and male flowers, respectively, in E. japonica; see Appendix S1: Table S1). At our study sites, E. emarginata tends to occur in coastal habitats and blooms from late autumn to early winter, whereas $E$. japonica is usually found in forests and blooms from late winter to early spring, with no overlap between the two species in flowering phenology.

\section{Hypothesis 1: effect of flower sex}

Preliminary work suggested that one major sexual difference in floral traits of E. emarginata and E. japonica was sugar concentration in nectar, which tended to be higher in female than male flowers (Appendix S1: Table S2, Fig. S1). The adaptive reason for this sexual difference, if any, remains uncertain, but one possibility is that female flowers have evolved to offer pollinators a better reward to compensate for their smaller size relative to males. In E. japonica, flower size is positively correlated with the number of insect visitors for a given amount of sugar in nectar (Tsuji and Ohgushi 2018). Despite this correlation, there is little difference in insect visitors between females and males (Tsuji and Ohgushi 2018), suggesting that female and male flowers may have evolved to attract pollinators using different floral traits, that is, a greater reward (more sugar) and a greater signal (larger size), respectively. Because high sugar concentration can increase the level of chemical stress that limits microbial survival and growth (Pozo et al. 2012, Vannette and Fukami 2014, Letten et al. 2018), we hypothesized that microbial abundance would be lower in female flowers. Sugar concentration might also affect insect visitation to flowers, but our previous study suggested that neither the frequency nor composition of insect visitors differed greatly between female and male flowers (Tsuji and Ohgushi 2018).
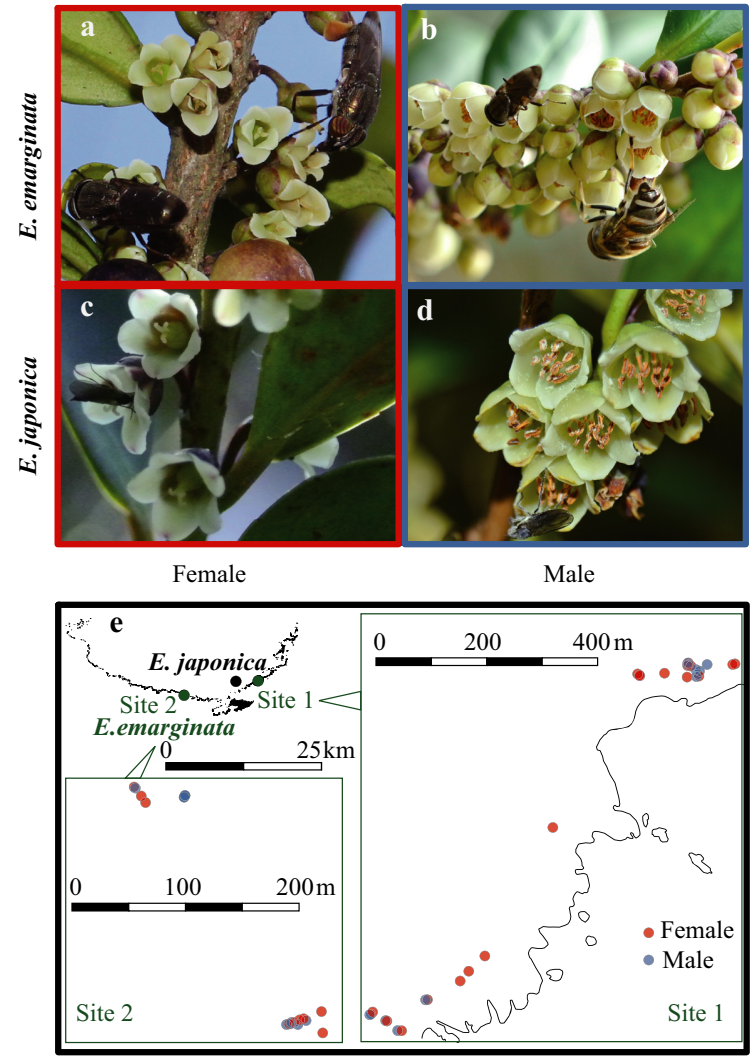

FIG. 1. Study system. (a) Female Eurya emarginata, visited by flies, Stomorhina obsoleta; (b) male E. emarginata, visited by $S$. obsoleta and a hoverfly, Eristalinus quinquestriatus; (c) female E. japonica, visited by a midge (Sciaridae); and (d) male E. japonica, visited by a tiny fly Rhamphomyia (Megacyttarus) brunneostriata. (e) Map of the study sites, where each dot indicates the location of a studied plant. Site $1,33^{\circ} 49^{\prime} 37^{\prime \prime} \mathrm{N}, 135^{\circ} 52^{\prime} 75^{\prime \prime} \mathrm{E}, 7 \mathrm{~m}$ above sea level, and site $2,33^{\circ} 29^{\prime} 32^{\prime \prime} \mathrm{N}, 135^{\circ} 40^{\prime} 2$ E, $14 \mathrm{~m}$ above sea level. Photo credit: K. Tsuji.

Thus, we expected sexual differences in nectar chemistry, rather than insect visitation, to be more important in determining nectar microbial abundance.

To test the hypothesis that microbial abundance is lower in female flowers (hypothesis 1), we took two approaches: nectar sampling and experimental inoculation. For nectar sampling, we collected nectar from wild plants and estimated microbial abundance and the concentration of chemical compounds, including sugars. We sampled nectar from a total of 1,736 open (not bagged) flowers on a total of 36 female and 37 male plants of the two species (Appendix S1: Table S3). Nectar was collected using 0.5 - or $5.0-\mu \mathrm{L}$ microcapillary tubes. The collected nectar was diluted in $40 \mu \mathrm{L}$ of autoclaved pure water (Merck Millipore, Burlington, MA, USA) and plated on fungal and bacterial agar plates on the day of nectar collection immediately after storage on ice and in a refrigerator. As in Vannette and Fukami (2017), we prepared fungal plates using yeast malt agar (YM) with $100 \mathrm{mg} / \mathrm{L}$ chloramphenicol (antibacterial) and bacterial plates using R2A agar supplemented with $20 \%$ sucrose and $100 \mathrm{mg} / \mathrm{L}$ cycloheximide (antifungal; see Appendix S1: Fig. S2). Plates were incubated at $25^{\circ} \mathrm{C}$ for $5 \mathrm{~d}$, and colony forming units counted. In our previous work (Peay et al. 
2012), we found that the number of colony-forming units (CFU) was correlated well with cell density in nectar, at least for several common species of nectar yeasts. We used the CFU data to estimate the abundance of fungi and bacteria that were culturable on the specific media, YM and R2A, respectively. Although not a good measure of total microbial abundance, the CFU data are still informative because many of the dominant taxa in floral nectar, as verified by cultureindependent methods (e.g., Vannette and Fukami 2017, Toju et al. 2018), which primarily include species of Metschnikowia and Acinetobacter, are culturable on the media we used.

To determine if the effect of flower sex was consistent across microbial species, we identified some of the microbial colonies (118 colonies from bacterial plates and 209 colonies from fungal plates; all bacterial isolates were taken from the R2A medium and all fungal isolates from the YM medium) to species by haphazardly selecting colonies and Sangersequencing them. For Sanger-sequencing, we plated nectar samples collected from total of 288 E. emarginata flowers in November 2016 (Appendix S1: Table S3). Species identification involved the same methods as in Vannette et al. (2013) (see Appendix S2).

To measure nectar quality of the plants that were used for the microbial analysis (some plants did not have a sufficient number of flowers, and we could not measure nectar quality for these plants), we used a portable refractometer (SK100R; Sato Keiryoki Manufacturing Company, Tokyo, Japan) to measure nectar Brix values, which would measure $\mathrm{g}$ sucrose in $100 \mathrm{~g}$ pure sucrose solution and could serve as a proxy for the dissolved solid (including sugars and amino acids) concentration in nectar. To supplement the Brix data, we also present sugar concentration data that we had previously measured using high-performance liquid chromatography (HPLC) applied to 77 E. japonica nectar samples from the Kozagawa site (see Appendix S1: Tables S2 and S3).

The purpose of the second approach, experimental inoculation, was to compare the growth of nectar-colonizing yeasts in female and male flowers. We inoculated wild E. japonica flowers with a strain of Metschnikowia reukaufii, one of M. gruessii, or both sequentially (See Appendix S2). These yeasts were the most commonly isolated fungal species from E. japonica (Appendix S1: Table S4). Each flower was inoculated with approximately 200 cells suspended in $0.5 \mu \mathrm{L} \mathrm{H} \mathrm{H}_{2} \mathrm{O}$. We used 19 pairs of female and male plants. On each plant, we enclosed a flower-bearing branch in a mesh bag (mesh size $0.4 \mathrm{~mm}$, which allowed microbes to enter if they arrived by themselves, but not via insects that could not access bagged flowers), which prevented most insects from accessing flowers. Bagging, however, did not entirely exclude insects, as the hover fly, Eristalinus quinquestriatus, could occasionally reach $E$. emerginata nectar through the mesh using their narrow and long proboscis. We tagged flowers on the bagged branch using different color strings for identification purposes. On each branch, we inoculated a newly opened flower with $M$. reukaufii, another new flower with M. gruessii, and another with $0.5 \mu \mathrm{L} \mathrm{H}_{2} \mathrm{O}$. We used 57 female flowers and 58 male flowers for this experiment (Appendix S1: Table S3). Four days after inoculation, nectar was collected from each flower using $0.5-\mu \mathrm{L}$ microcapillary tubes, diluted with $\mathrm{H}_{2} \mathrm{O}$, plated on $\mathrm{YM}$ media without chloramphenicol, and colonies counted the same way as described above.

To estimate the accuracy with which we could distinguish $M$. reukaufii and $M$. gruessii colonies, we analyzed 485 haphazardly chosen colonies from these plates by the restriction fragment length polymorphism (RFLP) analysis with Hae III restriction enzymes, following Peay et al.'s (2012) methods. Of the two colony morphology types that were noticeable on the plates, one was always M. reukaufii (274 colonies tested), whereas the other type (211 colonies tested) included 175 M. gruessii (83\%), 34 M. reukaufii (16\%), and 2 other species $(1 \%)$. Thus, we were able to count colonies of $M$. reukaufii and $M$. gruessii separately with 93\% (i.e., $(274+175) / 485$ colonies) accuracy.

\section{Hypothesis 2: interactive effect of flower sex and animal visitation}

Animal visitation can affect nectar microbes at least in two ways. First, many nectar-colonizing microbes rely on animals for dispersal from flower to flower (Belisle et al. 2012, De Vega and Herrera 2012, Schaeffer and Irwin 2014), so reduced animal visitation should result in a decline in the incidence and abundance of microbes in nectar. Second, flower visitors can also cause chemical changes to nectar by removing nectar from flowers (Canto et al. 2008). Without nectar removal by animals, dissolved substances including sugars would remain in the flower while more nectar is secreted. Consequently, chemical concentration in nectar can increase when floral morphology is such that nectar is exposed to air and therefore evaporates quickly, as in our plants (Fig. 1a-d). Heightened nectar concentration, which can exacerbate chemical stress, can then reduce microbial abundance in nectar. For these two reasons, we expected animal visitation to increase microbial abundance. However, because preliminary results for $E$. japonica indicated that nectar was inherently more sugar-rich in female flowers than male flowers, imposing high chemical stress on nectar microbes regardless of animal visitation, we hypothesized that animal visitation would have a greater effect on nectar microbes in male than in female flowers.

To test this hypothesis (hypothesis 2), we enclosed some flower buds in a small organza bag to prevent insects from accessing them. This experiment was done at the same time as nectar sampling for testing hypothesis 1 . Nectar of 8 bagged and 8 exposed (control) flowers were collected from each plant (Appendix S1: Table S3). The collected nectar was then used to estimate microbial abundance as above. In addition, we also used a sucrose refractometer to measure nectar Brix values in bagged and exposed flowers (Appendix S1: Table S3). To supplement the Brix measurements, we also used HPLC as above to analyze nectar collected from a total of 16 and 21 bagged and 15 and 24 exposed flowers on female and male E. japonica plants, respectively (Appendix S1: Table S3).

\section{Hypothesis 3: interactive effect of flower sex and dispersal history}

When flowers are visited by multiple insect individuals and when some individuals introduce only one yeast species, 
a) Fungal frequency, YM

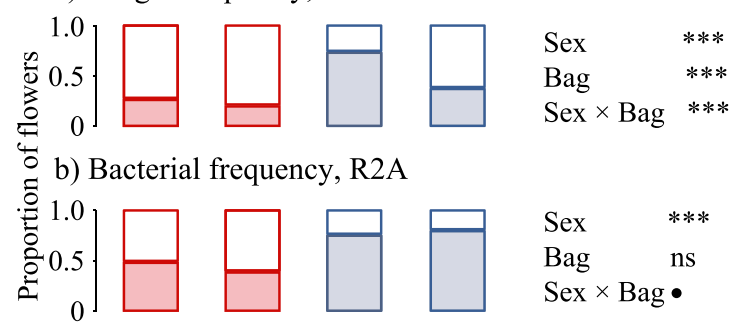

c) Fungal abundance, YM,

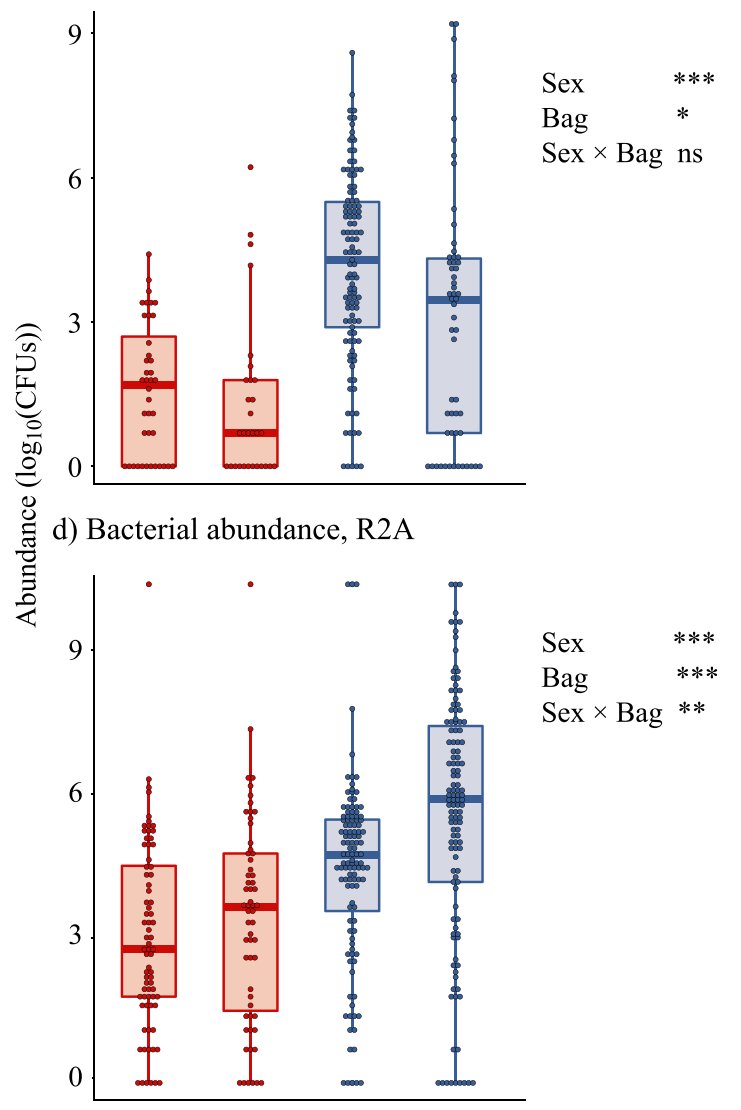

e) Nectar concentration

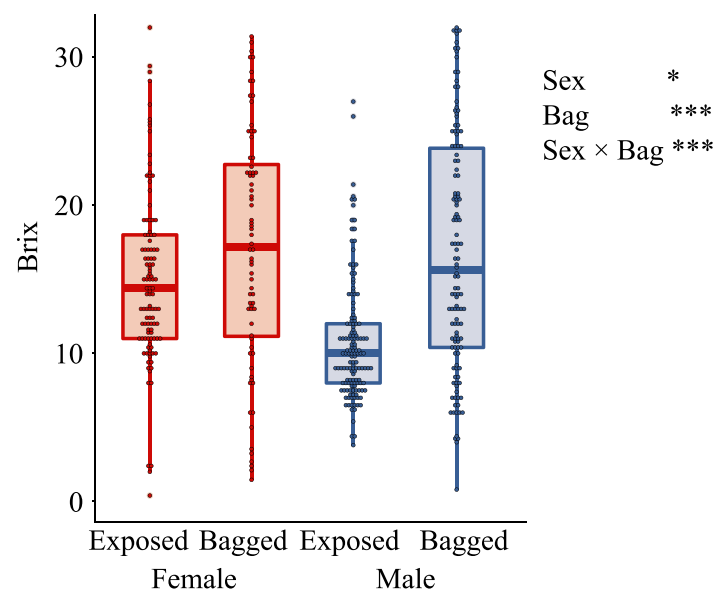

FIG. 2. Frequency and abundance of nectar microbes, and nectar concentration (estimated by the Brix value) in E. emarginata. (a) Frequency of fungal detection using YM media and (b) bacterial for example, $M$. reukaufii, and some only another, for example, M. gruessii, there can be substantial variation among flowers in dispersal history, or more specifically, in the order in which the yeast species are introduced by insect vectors. Previous research investigating the effect of dispersal history on nectar microbial communities has revealed strong priority effects, in which the order of microbial species arrival influences how species affect one another (Peay et al. 2012, Mittelbach et al. 2016). One general hypothesis that may apply to animals (Chase 2007), plants (Kardol et al. 2013, Leopold et al. 2015), and microbes (Vannette and Fukami 2014) is that priority effects are stronger in less stressful environments. Assuming lower chemical stress in male flowers, we hypothesized that priority effects would be stronger in male flowers than in female flowers.

To test this hypothesis (hypothesis 3), we inoculated wild, newly opened $E$. japonica flowers with both strains of $M$. reukaufii and $M$. gruessii used for testing hypothesis 1 in two different orders: (1) $M$. reukaufii on day 0 and $M$. gruessii on day 2 and (2) $M$. gruessii on day 0 and $M$. reukaufii on day 2 . We did the inoculation over multiple days to increase the number of replicates (flowers). On any given day of inoculation, the number of flowers we used was the same between the two inoculation-order treatments, and both treatments were applied on the same plants. Two days after the second inoculation, nectar was collected and plated as described above. The flowers were bagged during the experiment. We used 77 female flowers and 78 male flowers for this experiment (Appendix S1: Table S3).

\section{Statistical analysis}

To test hypotheses 1 and 2, we compared microbial abundance between female and male flowers and between those with and without bags, using a two-part model (Zuur et al. 2009). The first part tested whether the proportion of flowers in which microbes were detected differed between female and male flowers. We used a generalized linear mixed model (GLMM) with a binomial distribution and a logistic function in the lme4 package (Bates et al. 2015) and the Type II Wald chi-square test (Langsrud 2003) in the car package (Fox and Weisberg 2011) in R version 3.4.0 (R Core Team 2017). In this model, we used microbial detection (presence or absence) as the response variable; flower sex, bagging, and their interaction as fixed predictors; and plant and site ID as nested random effects. The second part focused on those flowers from which nectar microbes were detected. We used GLMM with a negative binomial distribution and a logistic function to analyze microbial abundance (CFU) as a function of the same fixed predictors and random effects as in the first model.

\section{(Fig. 2. Continued)}

detection using R2A media. Filled and open bars indicate flowers from which microbes were detected and those from which they were not, respectively. For both fungal and bacterial data, each of the four treatments had 152 flowers as replicates. (c) Fungal and (d) bacterial abundance. (e) Nectar concentration (Brix is a measure of $\mathrm{g}$ sucrose in $100 \mathrm{~g}$ pure sucrose solution; see also Appendix S1: Fig. S7). ${ }^{\bullet} P<0.1,{ }^{*} P<0.05,{ }^{*} P<0.01,{ }^{* * *} P<0.001$; ns, not significant. For the box plots (c)-(e), mid line, box edges, whiskers, points show median, lower or upper quartile, minimum or maximum, and row data points, respectively. 


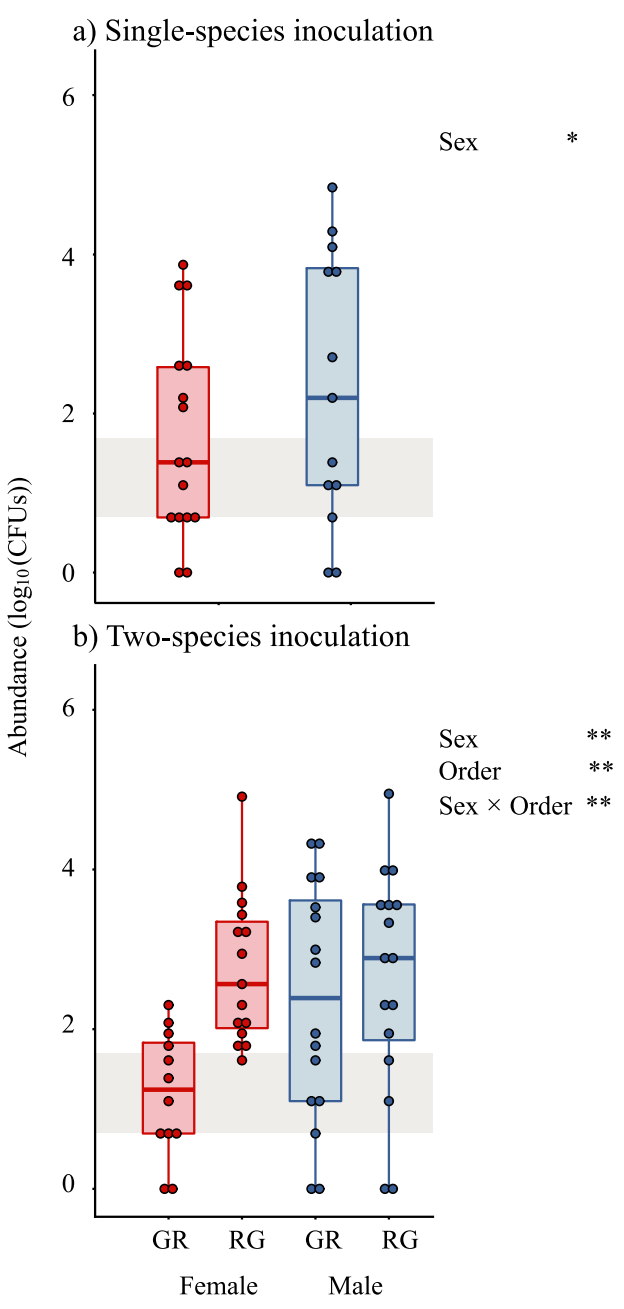

FIG. 3. Abundance of Metschnikowia gruessii (yeast) introduced to E. japonica in (a) single-species and (b) two-species inoculation experiments. $\mathrm{G}$ and $\mathrm{R}$ indicate inoculated species, $M$. gruessii and $M$. reukaufii, respectively. GR and $\mathrm{RG}$ represent inoculation order (GR, first inoculation was $G$ and second was R; RG, first was R and second was G). The shaded area indicates the estimated possible range of the initial abundance of viable cells (estimated as 10-100\% viability of inoculated cells). In this experiment, no yeast was detected from the control flowers. Asterisks, boxes, whiskers, and points are as in Fig. 2.

We used a similar two-part model to analyze nectar Brix values, with the same fixed predictors and random effects as above. The Brix value could not be measured from some of the flowers, either because too little nectar was collected or because the Brix value was too high, falling outside the measurable range. Both these reasons are likely to be associated with high nectar concentration. Thus, in the first GLMM, we used availability of the Brix value as the response variable. The second GLMM, with a gamma distribution, analyzed the Brix value as the response variable.

In addition, to more directly examine how the Brix value was related to $\mathrm{CFU}$, we counted the number of plants that fell into the following four categories: (1) those where bagging decreased Brix value and increased CFU (both values were averaged across flowers on the same host plant), (2) those where bagging increased both, (3) those where bagging increased Brix value and decreased CFU, and (4) those where bagging decreased both. We used a $\chi^{2}$ test to determine if the four categories differed in frequency and if the answer depended on flower sex.

Using the Sanger-sequence data from the two plant species, relative species frequency was analyzed by $\chi^{2}$ and Fisher's exact tests to determine if male and female flowers differed in species composition. For this test, we focused on three species (Acinetobacter boissieri, A. nectaris, and $M$. reukaufii) since they were isolated frequently from nectar (Appendix S1: Table S4). Most other species, we isolated from Eurya nectar (e.g., species of Fusarium, Cryptococcus, and Rhodotorula) were rare (Appendix S1: Table S4), and their biology suggests that they do not grow well in floral nectar (Brysch-Herzberg 2004).

To test hypothesis 1 with experimental inoculation data, we used a two-part model similar to the ones described above, with CFU of each species as the response variable and flower sex as a predictor. The two-part model we used to test hypothesis 3 with experimental inoculation had CFU as the response variable, and flower sex, inoculation order, and their interaction as fixed predictors. In both analyses, plant ID and inoculation date were included as random effects.

\section{RESUlts}

Microbes were found in the majority $(69 \%)$ of E. emarginata flowers (Fig. 2a, b), but rarely (7\%) detected in E. japonica flowers (Appendix S1: Fig. S3) unless experimentally introduced (Fig. 3; Appendix S1: Fig. S4). For this reason, we will primarily highlight results for E. emarginata and report results for E. japonica more briefly (presented more thoroughly in Supporting Information) in reference to hypotheses 1 and 2 . Incidentally, the paucity of naturally occurring microbes in E. japonica allowed for a rigorous test of hypothesis 3 on the effect of the experimentally introduced yeast strains on each other, which would have been difficult to interpret if naturally occurring microbes were common.

\section{Hypothesis 1: effect of flower sex}

In E. emarginata, both fungi (Fig. 2a; Appendix S1: Fig. S5) and bacteria (Fig. 2b) were detected more frequently in male than female flowers (overall, more than twice as frequent). Their abundances (Fig. 2c, d; Appendix S1: Fig. S5) were also greater in male flowers, with the median being more than 10 times as high (Appendix S1: Table S5). On average, male flowers contained more nectar than female flowers did (Appendix S1: Fig. S6), but even when the amount of nectar was taken into consideration, microbes were significantly more abundant in male flowers (Appendix S1: Table S6). In contrast, the nectar Brix value was higher in female flowers (Fig. 2e; Appendix S1: Table S7, Fig. S7). Similarly, in E. japonica, yeast frequency and bacterial and fungal abundance were greater in male flowers (Appendix S1: Table S5, Fig. S3), while nectar concentration was higher in female flowers (Appendix S1: Tables S2 and S7, Fig. S1). Female and male flowers also differed in species relative frequency (Table 1): female flowers were more likely to contain Acinetobacter boissieri than $A$. nectaris and $M$. reukaufii, and the opposite was true for male flowers $\left(\chi^{2}=44.4, P<0.01\right.$, Table 1$)$. In 
the inoculation experiment, $M$. reukaufii did not respond significantly to flower sex $\left(\chi^{2}=0.008, P=0.90\right.$, Appendix S1: Fig. S4), but $M$. gruessii was more abundant in male flowers $\left(\chi^{2}=4.11, P=0.04\right.$, Fig. 3a).

\section{Hypothesis 2: interactive effect of flower sex and animal visitation}

In $E$. emarginata, bagging decreased fungal frequency and abundance (Fig. 2a, c; Appendix S1: Fig. S4) and increased the Brix value (Fig. 2e; Appendix S1: Table S7, Fig. S7), and the effect on fungal frequency was greater in male than female flowers (Fig. 2a; Appendix S1: Table S5, Fig. S5), resulting in a significant negative association between fungal frequency and the Brix value on male, but not female, plants (Appendix S1: Table S8, Fig. S8a). In contrast, bagging had no significant effect on bacterial frequency (Fig. 2b) and increased bacterial abundance (Fig. 2d), which was positively associated with the Brix value (Appendix S1: Table S8, Fig. S8d). In E. japonica, we detected only small, mostly non-significant effects of bagging on microbial abundance (but see Appendix S1: Table S5, Fig. S3, showing bagging decreased bacterial frequency to an even lower level, suggesting that arthropods dispersed bacteria as well as fungi) and the Brix value (Appendix S1: Table S7).

\section{Hypothesis 3: interactive effect of flower sex and dispersal history}

In female E. japonica flowers, $M$. gruessii became more abundant when it was introduced after, rather than before,

TABLE 1. Number of female and male flowers from which dominant microbial species were detected.

\begin{tabular}{lrrrrr}
\hline \hline & \multicolumn{2}{c}{ Female } & & \multicolumn{2}{c}{ Male } \\
\cline { 2 - 3 } \cline { 6 - 6 } Species & No. & $\%$ & & No. & $\%$ \\
\hline Acinetobacter boissieri (bacterium) & 20 & 57 & & 19 & 11 \\
Acinetobacter nectaris (bacterium) & 1 & 3 & 51 & 29 \\
Metschnikowia reukaufii (yeast) & 14 & 40 & & 107 & 60 \\
Total & 35 & 100 & & 177 & 100 \\
\hline
\end{tabular}

M. reukaufii (Fig. 3b). In male flowers, neither species was affected by introduction order (Fig. 3b; Appendix S1: Tables S9 and S10, Fig. S4b).

\section{DisCUSSION}

Taken together, our results provide evidence that distinct communities develop in the nectar of female vs. male flowers. Consistent with hypothesis 1 , we found that fungi and bacteria that were culturable on YM and R2A media tended to attain a higher abundance in male flowers (Fig. 2a-d) and that fungi grew better in male E. japonica flowers (Fig. 3). Compared to the typical variation in nectar microbial community structure among plant species (e.g., De Vega et al. 2009, Herrera et al. 2009, Herrera and Pozo 2010, Álvarez-Pérez and Herrera 2013, Aleklett et al. 2014, Herrera 2014, Canto et al. 2015, Lievens et al. 2015, Mittelbach et al. 2015, Pozo et al. 2015), the intraspecific variation we report here was surprisingly large, with more than a 2- and 10 -fold difference between flower sexes in microbial prevalence (Fig. 2a, b) and abundance (Fig. 2c, d), respectively. In addition, the relative frequency of dominant microbial species differed between female and male flowers (Table 1) and yeast species responded differently to flower sex (Fig. 3; Appendix S1: Fig. S4), showing that sexual dimorphism in host plants can affect not just total abundance, but also species composition of microbial communities.

The mechanisms responsible for these effects remain unresolved and further experimental studies are needed to ascertain the exact mechanisms behind the findings. Nonetheless, our test of hypotheses 2 and 3 indicated some potential mechanisms. Namely, female and male plants appeared to differ in how animal-mediated changes in the chemical stress of nectar (hypothesis 2) and the dispersal history of microbes (hypothesis 3) influence the microbial communities. Consistent with hypothesis 2 , we saw a greater response of both fungi (Fig. 2a) and bacteria (Fig. 2d) in male than female flowers to experimental reduction in animal visitation. Reduced visitation probably led to both an increase in nectar concentration (Fig. 2e) and a decline in microbial dispersal, but nectar concentration might be more likely to underlie the results that support hypothesis 2 , as we

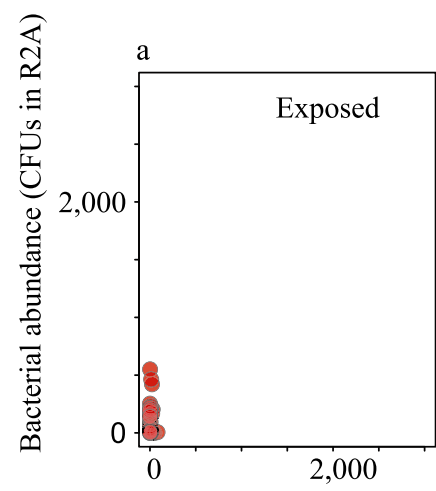

$\mathrm{b}$

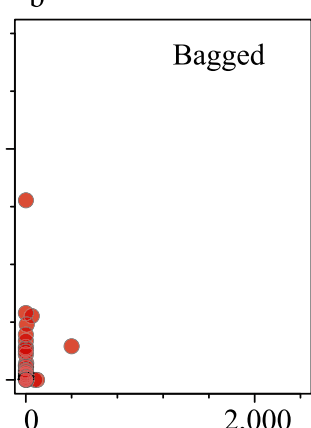

Fungal abundance (CFUs in YM)

$\mathrm{c}$
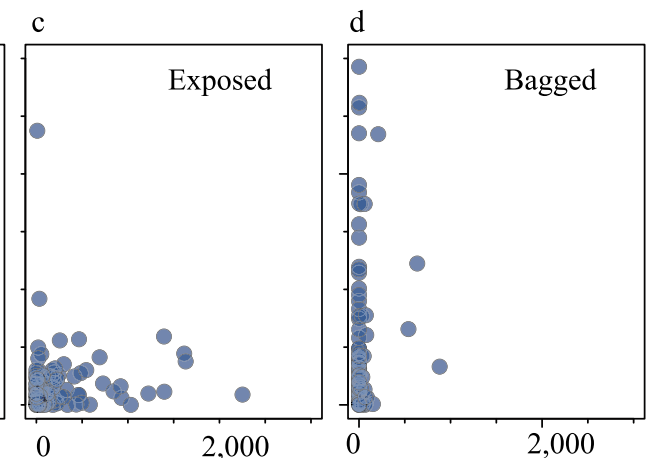

Male

FIG. 4. Association between bacteria and fungi in E. emarginata nectar. (a) Exposed female flowers, (b) bagged female flowers, (c) exposed male flowers, (d) bagged male flowers. See also Appendix S1: Fig. S9. 
observed a greater increase in nectar concentration in male flowers (Fig. 2e), whereas no obvious inherent difference in animal visitation was found between male and female flowers (Tsuji and Ohgushi 2018).

Reduced animal visitation caused a decline in yeast frequency and abundance (Fig. 2a, c; Appendix S1: Fig. S5), as expected from their reliance on animals for dispersal. In contrast, we did not see this effect for bacteria (Fig. 2b), and even observed an increase in their abundance as a result of reduced animal visitation (Fig. 2d). This result might suggest that reduced yeast dispersal by bagging caused competitive release of bacteria from yeasts (Tucker and Fukami 2014). Although we did not directly test for competitive release, the observed relationship between bacterial and fungal abundance is consistent with this possibility. Specifically, we saw no noticeable effect of bagging in female flowers, where yeasts were never common (Fig. 4a, b; Appendix S1: Fig. S9), while a large shift from yeast dominance (Fig. 4c; Appendix S1: Fig. S9) to bacterial dominance (Fig. 4d; Appendix S1: Fig. S9) was evident in male flowers. An additional line of evidence comes from E. japonica results. Bagging, which did not affect bacterial frequency in E. emarginata (Fig. 2b), decreased it in E. japonica (Appendix S1: Fig. S3), and the positive effect of bagging on bacterial abundance was observed only in E. emerginata (Fig. 2d), and not in E. japonica (Appendix S1: Fig. S3). The E. japonica results are what would be expected if there was no indirect effect of bagging on bacteria via yeasts. Consistent with this expectation, yeasts were almost non-existent in E. japonica (Appendix S1: Fig. S3). That so few nectar microbes were found in flowers of E. japonica, a late-winterblooming plant, might have to do with low ambient temperature, which slows down microbial growth, and the lack of co-flowering species from which nectar microbes can disperse. It is warmer and there are many other flowering species that can supply nectar microbes during $E$. emarginata's flowering season in late autumn. Other environmental factors that can affect nectar characteristics and insect vectors, including wind velocity, water availability, and soil fertility, may explain why the results differed between the two Eurya species, but we did not measure these factors.

The test of hypothesis 3 indicated that the effect of dispersal history depended on flower sex, though not as predicted. We expected a stronger effect in male flowers because we thought that lower nectar concentration in male flowers would make their nectar less harsh to microbes. Instead, we found a stronger effect of dispersal history in female flowers (i.e., M. gruessii abundance was $17 \%$ and $83 \%$ lower in female and male flowers, respectively, when $M$. gruessii was inoculated first than when it was inoculated later; see Fig. 3b). Hypothesis 3 assumes that priority effects are inhibitory (e.g., Vannette and Fukami 2014), but the priority effects we observed in this study appeared facilitative, with M. gruessii attaining higher abundance when introduced after $M$. reukaufii than introduced before it. The mechanism behind this facilitation is unknown, but one possibility is that $M$. reukaufii, which is particularly tolerant of high chemical stress (Pozo et al. 2012, Letten et al. 2018), reduced the level of stress as they grew in number, alleviating chemical stress for $M$. gruessii. This possible mechanism might explain why we saw priority effects only in female flowers, which had higher chemical concentrations compared to male flowers.

Although we did not examine the effect of nectar microbes on pollinator visitation in this study, previous studies suggest that bees and other flower visitors sometimes avoid nectar colonized by bacteria owing to microbeinduced changes in the chemical properties of nectar (Good et al. 2014, Junker et al. 2014, Vannette and Fukami 2014, 2016). In contrast, yeasts often have no or even positive effects on pollinator visitation (Herrera et al. 2013, Good et al. 2014, Schaeffer and Irwin 2014, Vannette and Fukami 2014). Complete understanding of the effects of plant sex on nectar microbial communities will likely require more experimental studies that establish causal relationships among host plants, their flower visitors including pollinators, and both the fungi and bacteria that colonize nectar.

The scope of research on the community-level effects of sexual dimorphism has been rather limited, with most studies either focusing on medically relevant animals or examining only one group of community members. Here, we have examined two functionally distinct groups, bacteria and fungi, both of which responded greatly to host plant sex, but also quite differently from each other. Although we examined only one case of host-associated communities in one specific part of the host in this study, sexual dimorphism is common across a range of plants and animals, and the communities associated with them are often functionally diverse. We suggest that sexual dimorphism may have more profound community-wide consequences than currently recognized.

\section{ACKNOWLEDGMENTS}

We thank Griselda Morales, Shin-ichi Nakano, Rika Ozawa, Jiro Sugisaka, Rie Shimizu-Inatsugi, and Junji Takabayashi for laboratory support, Noboru Katayama for advice on HPLC, Shohei Fujinaga for laboratory support and comments, and Callie Chappell, Manpreet Dhami, Po-Ju Ke, Andrew Letten, Avery Russell, Robert Schaeffer, Na Wei, two anonymous reviewers, and members of the community ecology group at Stanford University for comments. This work was supported by JSPS Grant-in Aid for JSPS fellows (25451) and for Young Scientist (40645280), the Kyoto University Foundation, and Stanford University's Office of International Affairs. K. Tsuji designed the study, conducted field and laboratory work, and analyzed data. K. Tsuji and T. Fukami wrote the paper.

\section{Literature Cited}

Ågren, J., K. Danell, T. Elmqvist, L. Ericson, and J. Hjältén. 1999. Sexual dimorphism and biotic interactions. Pages 217-246 in M. A. Geber, T. E. Dawson, and L. F. Delph, editors. Gender and sexual dimorphism in flowering plants. Springer, Berlin, Germany.

Aleklett, K., M. Hart, and A. Shade. 2014. The microbial ecology of flowers: an emerging frontier in phyllosphere research. Botany 92:253-266.

Álvarez-Pérez, S., and C. M. Herrera. 2013. Composition, richness and nonrandom assembly of culturable bacterial-microfungal communities in floral nectar of Mediterranean plants. FEMS Microbiology Ecology 83:685-699.

Bai, Y.-P., Y.-W. Zhang, R.-W. Gituru, J.-M. Zhao, and J.-D. Li. 2011. Sexual differences in reproductive characters and pollinator attractiveness in gynodioecious Glechoma longituba (Lamiaceae). Plant Species Biology 26:33-42.

Baker, H. G., and I. Baker. 1983. A brief historical review of the chemistry of floral nectar. Pages 126-152 in B. Bentley and T. S. 
Elias, editors. The biology of nectaries. Columbia University Press, New York, New York, USA.

Bates, D., M. Maechler, B. Bolker, and S. Walker. 2015. Fitting linear mixed-effects models using lme4. Journal of Statistical Software 67:1-48.

Belisle, M., K. G. Peay, and T. Fukami. 2012. Flowers as islands: spatial distribution of nectar-inhabiting microfungi among plants of Mimulus aurantiacus, a hummingbird-pollinated shrub. Microbial Ecology 63:711-718.

Bolnick, D. I., P. Amarasekare, M. S. Araújo, R. Bürger, J. M. Levine, M. Novak, V. H. W. Rudolf, S. J. Schreiber, M. C. Urban, and D. A. Vasseur. 2011. Why intraspecific trait variation matters in community ecology. Trends in Ecology and Evolution 26:183-192.

Brysch-Herzberg, M. 2004. Ecology of yeasts in plant-bumblebee mutualism in Central Europe. FEMS Microbiology Ecology 50:87-100.

Butler, M. A., S. A. Sawyer, and J. B. Losos. 2007. Sexual dimorphism and adaptive radiation in Anolis lizards. Nature 447:202-205.

Canto, A., C. M. Herrera, M. Medrano, R. Pérez, and I. M. García. 2008. Pollinator foraging modifies nectar sugar composition in Helleborus foetidus (Ranunculaceae): an experimental test. American Journal of Botany 95:315-320.

Canto, A., C. M. Herrera, I. M. García, M. García, and P. Bazaga. 2015. Comparative effects of two species of floricolous Metschnikowia yeasts on nectar. Anales del Jardín Botánico de Madrid 72:e019.

Chase, J. M. 2007. Drought mediates the importance of stochastic community assembly. Proceedings of the National Academy of Sciences USA 104:17430-17434.

Chung, M. G., and M. Y. Chung. 2000. Levels and partitioning of genetic diversity in populations of Eurya japonica and Eurya emarginata (Theaceae) in Korea and Japan. International Journal of Plant Sciences 161:699-704.

Cole, D. H., and T. L. Ashman. 2005. Sexes show differential tolerance to spittlebug damage and consequences of damage for multispecies interactions. American Journal of Botany 92:1708-1713.

Cong, X., W. Xu, S. Janton, W. A. Henderson, A. Matson, J. M. McGrath, K. Maas, and J. Graf. 2016. Gut microbiome developmental patterns in early life of preterm infants: impacts of feeding and gender. PLoS ONE 11:e0152751.

Cornelissen, T., and P. Stiling. 2005. Sex-biased herbivory: a metaanalysis of the effects of gender on plant - herbivore interactions. Oikos 111:488-500.

Costantini, D., E. Bruner, A. Fanfani, and G. Dell'Omo. 2007. Male-biased predation of western green lizards by Eurasian kestrels. Naturwissenschaften 94:1015-1020.

De Vega, C., and C. M. Herrera. 2012. Relationships among nectardwelling yeasts, flowers and ants: Patterns and incidence on nectar traits. Oikos 121:1878-1888.

De Vega, C., C. M. Herrera, and S. D. Johnson. 2009. Yeasts in floral nectar of some South African plants: Quantification and associations with pollinator type and sugar concentration. South African Journal of Botany 75:798-806.

Delph, L. F., and C. M. Lively. 1992. Pollinator visitation, floral display, and nectar production of the sexual morphs of a gynodioecious shrub. Oikos 63:161-170.

Eckhart, V. M. 1999. Sexual dimorphism in flowers and inflorescences. Pages 123-148 in M. A. Geber, T. E. Dawson, and L. F. Delph, editors. Gender and sexual dimorphism in flowering plants. Springer, Berlin, Germany.

Fierer, N., M. Hamady, C. L. Lauber, and R. Knight. 2008. The influence of sex, handedness, and washing on the diversity of hand surface bacteria. Proceedings of the National Academy of Sciences USA 105:17994-17999.

Fox, J., and S. Weisberg. 2011. An R companion to applied regression. Second edition. Sage, Thousand Oaks, California, USA.

Golonka, A. M., and R. Vilgalys. 2013. Nectar inhabiting yeasts in Virginian populations of Silene latifolia (Caryophyllaceae) and coflowering species. American Midland Naturalist 169:235-258.

Good, A. P., M.-P. L. Gauthier, R. L. Vannette, and T. Fukami 2014. Honey bees avoid nectar colonized by three bacterial species, but not by a yeast species, isolated from the bee gut. PLoS ONE 9:e86494.

Heil, M. 2011. Nectar: generation, regulation and ecological functions. Trends in Plant Science 16:191-200.

Herrera, C. M. 2014. Population growth of the floricolous yeast Metschnikowia reukaufii: effects of nectar host, yeast genotype, and host $\times$ genotype interaction. FEMS Microbiology Ecology $88: 250-257$

Herrera, C. M., and M. I. Pozo. 2010. Nectar yeasts warm the flowers of a winter-blooming plant. Proceedings of the Royal Society B 277:1827-1834.

Herrera, C. M., C. de Vega, A. Canto, and M. I. Pozo. 2009. Yeasts in floral nectar: a quantitative survey. Annals of Botany 103:1415-1423.

Herrera, C. M., M. I. Pozo, and M. Medrano. 2013. Yeasts in nectar of an early-blooming herb: sought by bumble bees, detrimental to plant fecundity. Ecology 94:273-279.

Junker, R. R., T. Romeike, A. Keller, and D. Langen. 2014. Densitydependent negative responses by bumblebees to bacteria isolated from flowers. Apidologie 45:467-477.

Kardol, P., L. Souza, and A. T. Classen. 2013. Resource availability mediates the importance of priority effects in plant community assembly and ecosystem function. Oikos 122:84-94.

Kwieciński, Z., Z. M. Rosin, Ł. Dylewski, and P. Skórka. 2017. Sexual differences in food preferences in the white stork: an experimental study. Science of Nature 104:39.

Lachance, M. A., W. T. Starmer, C. A. Rosa, J. M. Bowles, J. S. Barker, and D. H. Janzen. 2001. Biogeography of the yeasts of ephemeral flowers and their insects. FEMS Yeast Research 1:1-8.

Lajoie, G., and M. Vellend. 2015. Understanding context dependence in the contribution of intraspecific variation to community trait-environment matching. Ecology 96:2912-2922.

Langsrud, Ø. 2003. ANOVA for unbalanced data: Use type II instead of type III sums of squares. Statistics and Computing 13:163-167.

Leopold, D. R., A. J. Tanentzap, W. G. Lee, P. B. Heenan, and T. Fukami. 2015. Evolutionary priority effects in New Zealand alpine plants across environmental gradients. Journal of Biogeography 42:729-737.

Letten, A. D., M. K. Dhami, P. J. Ke, and T. Fukami. 2018. Species coexistence through simultaneous fluctuation-dependent mechanisms. Proceedings of the National Academy of Sciences USA 115:6745-6750

Lievens, B., J. E. Hallsworth, M. I. Pozo, Z. B. Belgacem, A. Stevenson, K. A. Willems, and H. Jacquemyn. 2015. Microbiology of sugar-rich environments: diversity, ecology and system constraints. Environmental Microbiology 17:278-298.

Markle, J. G., D. N. Frank, S. Mortion-Toth, C. E. Robertson, L. M. Feazel, U. Rolle-Kampczyk, M. Bergen, K. D. McCoy, A. J. Macpherson, and J. Danska. 2013. Sex differences in the gut microbiome drive hormone-dependent regulation of autoimmunity. Science 339:1084-1088.

Mittelbach, M., A. M. Yurkov, D. Nocentini, M. Nepi, M. Weigend, and D. Begerow. 2015. Nectar sugars and bird visitation define a floral niche for basidiomycetous yeast on the Canary Islands. BMC Ecology 15:2.

Mittelbach, M., A. M. Yurkov, R. Stoll, and D. Begerow. 2016. Inoculation order of nectar-borne yeasts opens a door for transient species and changes nectar rewarded to pollinators. Fungal Ecology 22:90-97.

Nepi, M., C. Soligo, D. Nocentini, M. Abate, M. Guarnieri, G. Cai, L. Bini, M. Puglia, L. Bianchi, and E. Pacini. 2012. Amino acids and protein profile in floral nectar: much more than a simple reward. Flora 207:475-481.

Peay, K. G., M. Belisle, and T. Fukami. 2012. Phylogenetic relatedness predicts priority effects in nectar yeast communities. Proceedings of the Royal Society B 279:749-758.

Petry, W. K., K. Perry, A. Fraemgen, S. K. Rudeen, M. Lopez, J. Dryburgh, and K. A. Moodey. 2013. Mechanisms underlying plant sexual dimorphism in multi-trophic arthropod communities. Ecology 94:2055-2065. 
Pozo, M. I., M.-A. Lachance, and C. M. Herrera. 2012. Nectar yeasts of two southern Spanish plants: the role of immigration and physiological traits in community assembly. FEMS Microbiology Ecology 80:281-293.

Pozo, M. I., C. M. Herrera, W. V. den Ende, K. Verstrepen, B. Lievens, and H. Jacquemyn. 2015. The impact of nectar chemical features on phenotypic variation in two related nectar yeasts. FEMS Microbiology Ecology 91:fiv055.

R Core Team. 2017. R: A language and environment for statistical computing. R Foundation for Statistical Computing, Vienna, Austria. https://www.R-project.org/

Ryan, M. J., M. D. Tuttle, and S. Rand. 1982. Bat predation and sexual advertisement in a neotropical anuran. American Naturalist 119:136-139.

Sakashita, H. 1992. Sexual dimorphism and food habits of the clingfish, Diademichthys lineatus, and its dependence on host sea urchin. Environmental Biology of Fishes 34:95-101.

Schaeffer, R. N., and R. E. Irwin. 2014. Yeasts in nectar enhance male fitness in a montane perennial herb. Ecology 95:1792-1798.

Schoener, T. W. 1967. The ecological significance of sexual dimorphism in size in the lizard Anolis conspersus. Science 155:474 477.

Sommer, S. 2000. Sex-specific predation on a monogamous rat, Hypogeomys antimena (Muridae: Nesomyinae). Animal Behavior 59:1087-1094.

Suzuki, Y., et al. 2017. Association between yogurt consumption and intestinal microbiota in healthy young adults differs by host gender. Frontiers in Microbiology 8:847.

Sylvia, K. E., C. P. Jewell, N. K. Rendon, E. A. S. John, and G. E. Demas. 2017. Sex-specific modulation of the gut microbiome and behavior in Siberian hamsters. Brain, Behavior, and Immunity 60:51-62.

Temeles, E. J., C. R. Koulouris, S. E. Sander, and W. J. Kress. 2009. Effect of flower shape and size on foraging performance and trade-offs in a tropical hummingbird. Ecology 90:1147-1161.

Tobler, M., C. M. Franssen, and M. Plath. 2008. Male-biased predation of a cave fish by a giant water bug. Naturwissenschaften 95:775-779.

Toju, H., R. L. Vannette, M. P. L. Gauthier, M. K. Dhami, and T. Fukami. 2018. Priority effects can persist across floral generations in nectar microbial metacommunities. Oikos 127:345-352.

Tsuji, K., and T. Ohgushi. 2018. Florivory indirectly decreases the plant reproductive output through changes in pollinator attraction. Ecology and Evolution 8:2993-3001.
Tsuji, K., and T. Sota. 2010. Sexual differences in flower defense and correlated male-biased florivory in a plant-florivore system. Oikos 119:1848-1853.

Tsuji, K., and T. Sota. 2011. Geographic variation in oviposition preference for male and female host plants in a geometrid moth: implications for evolution of host choice. Entomologia Experimentalis et Applicata 141:178-184.

Tsuji, K., and T. Sota. 2013. Florivores on the dioecious shrub Eurya japonica and the preferences and performances of two polyphagous geometrid moths on male and female plants. Entomological Science 16:291-297.

Tucker, C. M., and T. Fukami. 2014. Environmental variability counteracts priority effects to facilitate species coexistence: evidence from nectar microbes. Proceedings of the Royal Society B 281:20132637.

Vannette, R. L., and T. Fukami. 2014. Historical contingency in species interactions: towards niche-based predictions. Ecology Letters 17:115-124.

Vannette, R. L., and T. Fukami. 2016. Nectar microbes can reduce secondary metabolites in nectar and alter effects on nectar consumption by pollinators. Ecology 97:1410-1419.

Vannette, R. L., and T. Fukami. 2017. Dispersal enhances beta diversity in nectar microbes. Ecology Letters 20:901-910.

Vannette, R. L., M. P. L. Gauthier, and T. Fukami. 2013. Nectar bacteria, but not yeast, weaken a plant-pollinator mutualism. Proceedings of the Royal Society B 280:20122601.

Vesakoski, O., S. Merilaita, and V. Jormalainen. 2008. Reckless males, rational females: dynamic trade-off between food and shelter in the marine isopod Idotea balthica. Behavioural Processes 79:175-181.

Violle, C., B. J. Enquist, B. J. McGill, L. Jiang, C. H. Albert, C. Hulshof, V. Jung, and J. Messier. 2012. The return of the variance: intraspecific variability in community ecology. Trends in Ecology and Evolution 27:244-252.

Wagner, C. E., L. J. Harmon, and O. Seehausen. 2012. Ecological opportunity and sexual selection together predict adaptive radiation. Nature 487:36-370.

Wei, N., and T. L. Ashman. 2018. The effects of host species and sexual dimorphism differ among root, leaf and flower microbiomes of wild strawberries in situ. Scientific Reports 8:5195.

Zhang, M., and F. He. 2017. Plant sex affects the structure of plantpollinator networks in a subtropical forest. Oecologia 184:269-279.

Zuur, A. F., E. N. Ieno, N. J. Walker, A. A. Saveliev, and G. M. Smith. 2009. Mixed effects models and extensions in ecology with R. Springer-Verlag, New York, New York, USA.

\section{SUPPORTING INFORMATION}

Additional supporting information may be found in the online version of this article at http://onlinelibrary.wiley.com/doi/10.1002/ecy. 2494/suppinfo

\section{Data Availability}

Sequences available from the DNA Data Bank of Japan (DDBJ) at http://getentry.ddbj.nig.ac.jp under accession numbers LC333458LC333551; data are available from the Dryad Digital Repository at https://doi.org/10.5061/dryad.53vt348. 